Paper published as:

Nastro, V. \& Tancredi, U. (2010), 'Great Circle Navigation with Vectorial Methods', Journal of Navigation 63(3), 557--563.

DOI: 10.1017/S0373463310000044 


\title{
Great Circle Navigation with Vectorial Methods
}

\author{
Vincenzo Nastro and Urbano Tancredi \\ (University of Naples “Parthenope”, Italy)
}

The present paper is concerned with the solution of a series of practical problems relevant to great circle navigation, including the determination of the true course at any point on the great circle route and the determination of the lateral deviation from a desired great circle route. Intersection between two great circles or between a great circle and a parallel is also analyzed. These problems are approached by means of vector analysis, which yields solutions in a very compact form that can be computed numerically in a very straightforward manner. This approach is thus particularly appealing for performing computer-aided great circle navigation.

\section{KEY WORDS}

\section{Great circle 2. Vectors 2. Navigation}

1. INTRODUCTION. In air navigation it is well known that the Earth can be regarded as a sphere and, as a consequence, the shortest distance between any two points on its surface is an arc of a great circle. Great circles are obtained by the intersection with the surface of the Earth of any plane passing through the Earth's centre. For short distances, the difference between the great circle and the rhumb line is negligible. However, flying on a great circle allows saving considerable distance particularly on a long-range flight in high latitudes. For instance, the distance between London and Tokyo is about 6100 n.m. by rhumb line and 5170 n.m. by great circle, which allows saving about 930 n.m. 
Nevertheless, unlike the rhumb line that crosses all meridians at the same angle, the angle between a great circle route and the meridians constantly changes as progress is made along the route and is different at every point along the great circle. This implies that a vehicle shall be continuously steered to follow a great circle route. This necessity gives rise to a series of problems to be solved, such as: the determination of the True Course (TC), that is, the angle between the great circle route and the meridians, at any point on the great circle route; the determination of the lateral deviation, or Cross Track Distance (XTK), from a desired great circle route; and the intersection between two great circles or between a great circle and a parallel.

The position of a point $\mathrm{P}$ on the Earth's surface of latitude $\varphi$ and longitude $\lambda$ can be represented on a unit sphere by the unit vector $\mathbf{P}$ joining the Earth's centre to the point itself. The $\mathbf{P}$ vector has components given by:

$$
\mathbf{P} \equiv(\cos \varphi \cos \lambda, \quad \cos \varphi \sin \lambda, \quad \sin \varphi)
$$

with reference to an ECEF (Earth Centred Earth Fixed) frame: a right-handed, orthonormal coordinate system whose origin is located at the Earth's centre and has axes fixed to the Earth. Its z-axis points towards the north pole along the spin axis of the Earth and its $\mathrm{x}$-axis is the intersection of the reference meridian with the equator.

Therefore, great circle navigation can be developed taking advantage of vector analysis, allowing a continuous control of the trajectory and the solution of more complex problems such as the previously mentioned ones. Solutions obtained applying vector analysis to great circle navigation problems have a very compact form, and can be computed numerically in a very straightforward manner. This approach is thus particularly appealing for performing computer-aided great circle navigation. 
This problem has been already examined in a previous paper of the first author (Nastro, 2000), and recently discussed in this Journal (Earle, 2005; Tseng and Lee, 2007); the present manuscript reports some of this previous paper's results in a more compact form.

2. EQUATION OF THE GREAT CIRCLE. Figure 1 shows the great circle between the departure point $P_{1}\left(\varphi_{1}, \lambda_{1}\right)$ and the arrival point $P_{2}\left(\varphi_{2}, \lambda_{2}\right)$, where $\theta$ stands for the shortest distance between these two points. For the sake of simplicity, the meridian passing through $P_{1}$ is taken as the reference meridian, implying that the components of the vectors $\mathbf{P}_{1}$ and $\mathbf{P}_{2}$ are:

$$
\begin{aligned}
& \mathbf{P}_{1} \equiv\left(\begin{array}{lll}
\cos \varphi_{1} & 0 & \sin \varphi_{1}
\end{array}\right) \\
& \mathbf{P}_{2} \equiv\left(\begin{array}{lll}
\cos \varphi_{2} \cos \Delta \lambda & \cos \varphi_{2} \sin \Delta \lambda & \sin \varphi_{2}
\end{array}\right)
\end{aligned}
$$

where $\Delta \lambda=\lambda_{2}-\lambda_{1}$ is the difference of longitude between such points.

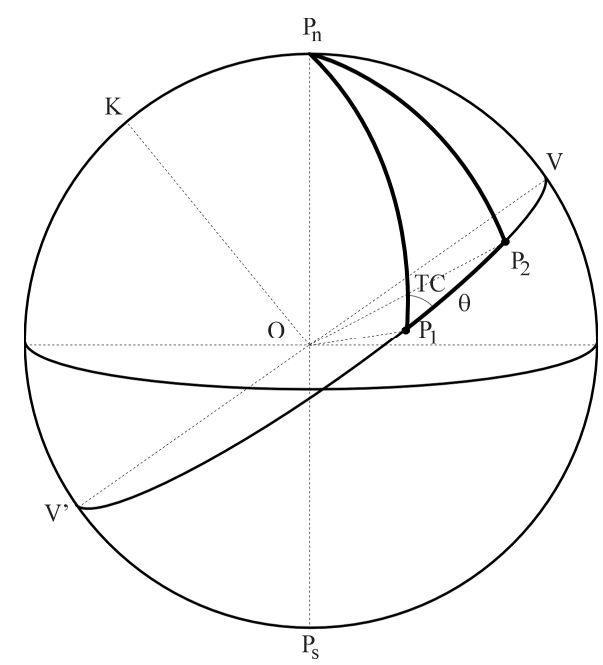

Figure 1. Representation of the great circle 
The distance $\theta$ between the two given points $P_{1}$ and $P_{2}$ is given from the dot product between the two corresponding vectors:

$$
\theta=\cos ^{-1}\left(\mathbf{P}_{\mathbf{1}} \cdot \mathbf{P}_{\mathbf{2}}\right)
$$

The vector $\mathbf{K}$, representing the great circle pole, can be obtained normalizing to one the vector cross product between $\mathbf{P}_{1}$ and $\mathbf{P}_{2}$, as:

$$
\mathbf{K}=\frac{\mathbf{P}_{\mathbf{1}} \times \mathbf{P}_{\mathbf{2}}}{\sin \theta}
$$

The coordinates of the vertex $\mathrm{V}$ of the hemisphere of interest (e.g. the northern one in Figure 1), that is, the point on the great circle path that is nearest to the geographic pole, can be obtained using the latitude and the longitude of the great circle pole $\left(\lambda_{K}, \varphi_{K}\right)$, as follows:

$$
\varphi_{V}=90^{\circ}-\varphi_{K} \quad ; \quad \lambda_{V}=\lambda_{K} \pm 180^{\circ}
$$

From Figure 2, $\mathbf{P}_{\mathbf{2}}$ can be seen as the result of a rotation of the vector $\mathbf{P}_{\mathbf{1}}$ around the direction $\mathbf{K}$ of an angle $\theta$ :

$$
\mathbf{P}_{\mathbf{2}}=\cos \theta \mathbf{P}_{\mathbf{1}}+\sin \theta\left(\mathbf{K} \times \mathbf{P}_{\mathbf{1}}\right)
$$

Analogously, for a generic point $\mathrm{P}$ on the great circle at a distance $\theta_{1}=k \theta$ from $P_{l}$ (where $k \in[0,1])$ the following holds:

$$
\mathbf{P}=\cos \theta_{1} \mathbf{P}_{\mathbf{1}}+\sin \theta_{1}\left(\mathbf{K} \times \mathbf{P}_{\mathbf{1}}\right)
$$




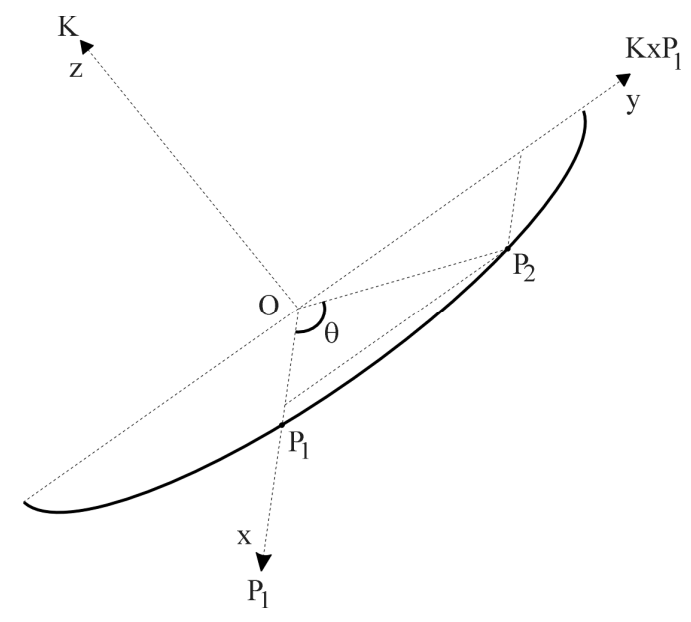

Figure 2. Rotation of the vector $\mathrm{P}_{1}$ of an angle $\theta$

The vector $\mathbf{K} \times \mathbf{P}_{\mathbf{1}}$ can be expressed in terms of the two vectors $\mathbf{P}_{\mathbf{1}}$ and $\mathbf{P}_{\mathbf{2}}$, making use of equation (4), yielding :

$$
\mathbf{P}=\cos \theta_{1} \mathbf{P}_{\mathbf{1}}+\sin \theta_{1} \mathbf{T}_{1}
$$

where the vector $\mathbf{T}_{\mathbf{1}} \equiv\left(T_{l x}, T_{l y}, T_{l z}\right)=\left(\mathbf{P}_{\mathbf{2}}-\cos \theta \mathbf{P}_{\mathbf{1}}\right) / \sin \theta$ is orthogonal to $\mathbf{P}_{\mathbf{1}}$ and tangent to the great circle at the departure point $\mathrm{P}_{1}$.

The relation (5) can be regarded as the equation of the great circle; for instance, if $\theta_{1}=k \theta=0.5 \theta$ the vector $\mathbf{P}$ is relative to the mid-point of the great circle.

The components of the vector $\mathbf{T}_{1}$ can be expressed in terms of latitude and True Course at the departure point $\mathrm{P}_{1}, \mathrm{TC}_{1}$, by applying the sine and the "four part" formulas to spherical triangle $P_{n} P_{1} P_{2}$ of Figure 1, yielding:

$$
\mathbf{T}_{1} \equiv\left(\begin{array}{lll}
-\sin \varphi_{1} \cos T C_{1} & \sin T C_{1} \quad \cos \varphi_{1} \cos T C_{1}
\end{array}\right)
$$

In case the determination of the TC at the departure point $\mathrm{P}_{1}$ is of interest, equation (6) can be exploited to obtain: 


$$
T C=\tan ^{-1}\left(T_{1 y} \cos \varphi_{1} / T_{1 z}\right)
$$

The above results are easily extended for determining the TC at any point $P$ of the great circle route, by considering the corresponding vector $\mathbf{P}$ in place of $\mathbf{P}_{\mathbf{1}}$ in obtaining equation (7).

3. DETERMINATION OF THE CROSS TRACK DISTANCE. In air navigation it is necessary to perform a continuous comparison between the present position derived from the airborne navigation system and the desired position on the great circle.

The deviation of the current navigation fix $\mathrm{P}$ from the great circle is represented in Figure 3 by the length of the $\operatorname{arc} \mathrm{PP}_{0}$, where $\mathrm{P}_{0}$ is the closest point of the great circle to $\mathrm{P}$ : the distance $\mathrm{PP}_{0}$ is known as XTK (Cross Track Distance).

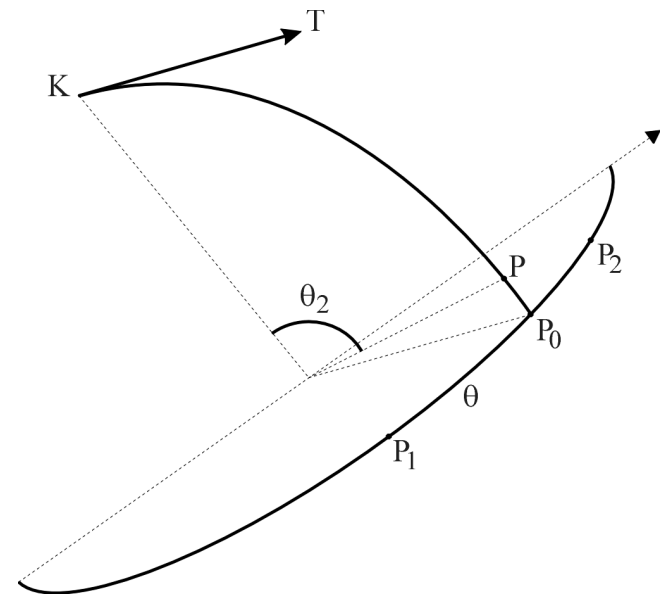

Figure 3. Determination of the Cross Track Distance (XTK)

The coordinates of the point $\mathrm{P}_{0}$ are derived from the vector $\mathbf{P}_{\mathbf{0}}$ that coincides with the vector $\mathbf{T}$ relative to the great circle between $\mathrm{K}$ and $\mathrm{P}$ : 


$$
\mathbf{P}_{\mathbf{0}}=\frac{\mathbf{P}-\cos \theta_{2} \mathbf{K}}{\sin \theta_{2}}
$$

where:

$$
\theta_{2}=\cos ^{-1}(\mathbf{K} \cdot \mathbf{P})
$$

At last, noting that the Cross Track Distance is complementary to $\theta_{2}$, it can be computed as $X T K=90^{\circ}-\theta_{2}$.

The availability of the above explicit expression for computing the cross track distance is beneficial for tracking the desired great circle route. In air navigation, for instance, the cross track distance can be coupled to a flight guidance computer that keeps the aircraft on the great circle course by issuing commands based on the current XTK value, computed as previously shown.

4. INTERSECTION OF TWO GREAT CIRCLES. Figure 4 represents two great circles: the first one connecting the points $\mathrm{P}_{1}$ and $\mathrm{P}_{2}$, whose pole is $\mathrm{K}_{1}$, and the second one between $\mathrm{P}_{3}$ and $\mathrm{P}_{4}$ with pole $\mathrm{K}_{2}$. The intersection point I can be derived from the vector $\mathbf{I}$ that is orthogonal to the vectors $\mathbf{K}_{\mathbf{1}}$ and $\mathbf{K}_{2}$; consequently:

$$
\mathbf{I} \cdot\left(\mathbf{K}_{1}-\mathbf{K}_{2}\right)=0
$$




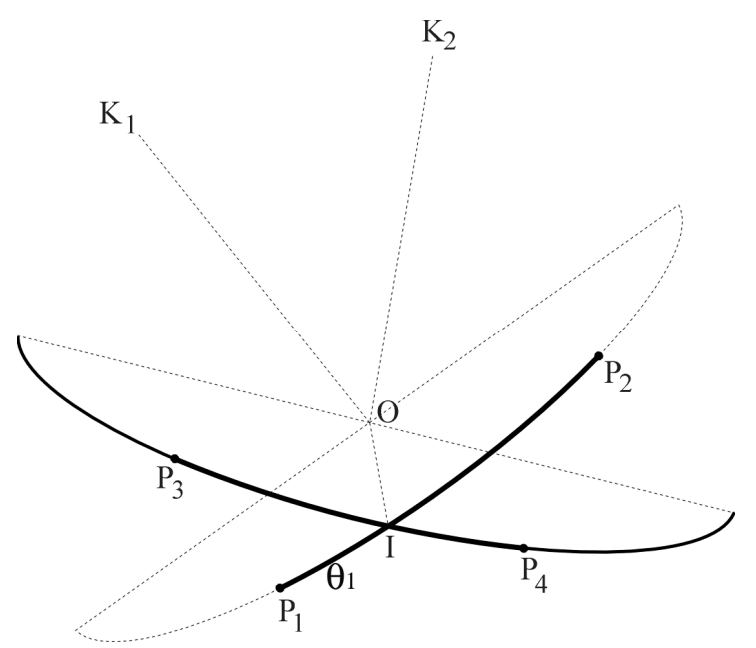

Figure 4. Intersection of two great circles

The vector $\mathbf{I}$ can be related by equation (5) to the known vectors $\mathbf{P}_{\mathbf{1}}, \mathbf{T}_{\mathbf{1}}$, and to the unknown distance $\theta_{1}$ between $\mathrm{P}_{1}$ and I. Substituting in the above equation, remembering that the dot product of orthogonal vectors is zero, and rearranging, yields the following expression for determining $\theta_{1}$, and, consequently, the intersection point I.

$$
\theta_{1}=\tan ^{-1}\left(-\frac{\mathbf{P}_{\mathbf{1}} \cdot \mathbf{K}_{\mathbf{2}}}{\mathbf{T}_{\mathbf{1}} \cdot \mathbf{K}_{\mathbf{2}}}\right)
$$

The above equation can be made specific in particular cases of interest, such as the intersection between a great circle and a meridian of longitude $\lambda_{m}$, or between a great circle and the equator, by setting:

$$
\begin{aligned}
& K_{2} \equiv\left(\sin \Delta \lambda_{m}, \quad-\cos \Delta \lambda_{m}, \quad 0\right) \text { for the meridian } \\
& K_{2} \equiv(0,0,1) \quad \text { for the equator }
\end{aligned}
$$


5. INTERSECTION OF THE GREAT CIRCLE WITH A PARALLEL. The coordinates of the intersection point $\mathrm{I}_{1}$ between a great circle and a parallel can be determined by the knowledge of the distance $\theta_{1}$ between the departure point $\mathrm{P}_{1}$ and $\mathrm{I}_{1}$ (Figure 5).

The vector $\mathbf{I}_{1}$ can be obtained by the rotation of the vector $\mathbf{P}_{1}$ around $\mathbf{K}_{1}$ until:

$$
\mathbf{I}_{1} \cdot \mathbf{K}_{2}=\sin \varphi_{p}
$$

where $\mathbf{K}_{2}$ is coincident with the unit vector $\mathbf{k} \equiv(0,0,1)$ and $\varphi_{p}$ stands for the latitude of the parallel. From relation (5) we have:

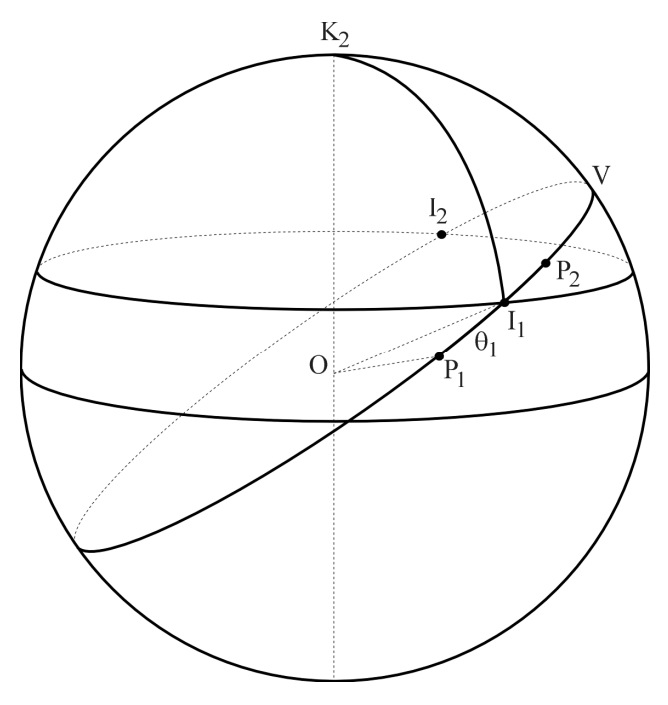

Figure 5. Intersection of the great circle with a parallel

$$
\left(\cos \theta_{1} \mathbf{I}_{1}+\sin \theta_{1} \mathbf{T}_{1}\right) \cdot \mathbf{K}_{2}=\sin \varphi_{p}
$$

or, equivalently:

$$
\cos \theta_{1} \sin \varphi_{p}+T_{1 z} \sin \theta_{1}=\sin \varphi_{p}
$$


This relation can be written as:

$$
a \cos \theta_{1}+b \sin \theta_{1}=c
$$

Expressing $\sin \theta_{1}$ and $\cos \theta_{1}$ in terms of the tangent of $\theta_{1}$, the above equation becomes quadratic in $\tan \theta_{1}$, whose solution is:

$$
\theta_{1}=\tan ^{-1}\left[\frac{-a b \mp c \sqrt{a^{2}+b^{2}-c^{2}}}{b^{2}-c^{2}}\right]
$$

In case the discriminant is positive, we have two distinct and real roots $\left(\varphi_{p}<\varphi_{v}\right)$, if it is zero we have a double real root $\left(\varphi_{p}=\varphi_{v}\right)$, whereas when the discriminant is negative there are no real roots $\left(\varphi_{p}>\varphi_{v}\right)$.

6. CONCLUSION. This paper has presented results for several practical problems relevant to navigation along a great circle route making use of vector analysis. These results are given in a compact form that is suitable for numerical implementation, thus being particularly appealing for computer-aided great circle navigation.

\section{REFERENCES}

1. Nastro, V. (2000), Problemi di navigazione ortodromica risolti con notazioni vettoriali, Studi in memoria di Antonino Sposito, 37-47.

2. Earle, M.A. (2005). Vector solutions for great circle navigation. This Journal, 58, $451-457$. 
3. Tseng, W.K. and Lee, H.S. (2007). The vector function for distance travelled in great circle navigation. This Journal, 60, 150-164. 